\title{
Functional outcome and quality of life after angiography and operation for unruptured intracranial aneurysms
}

Theodora W M Raaymakers, on behalf of the MARS Study Group (Members of the MARS Study Group are listed in the appendix)

\begin{abstract}
Objectives-To assess outcome after elective treatment for unruptured intracranial aneurysms.

Methods-Of 193 consecutive patients with subarachnoid haemorrhage 626 first degree relatives (parents, siblings, children) were screened with magnetic resonance angiography. Subsequently, 18 relatives underwent elective angiography and operation. Outcome was assessed in terms of impairments (neurological examination), disabilities (Barthel index), handicaps (Rankin scale), and quality of life (sickness impact profile (SIP) and short form-36 (SF-36)) 3 months and 1 year after operation; it was compared with baseline measurements.

Results-Before angiography all patients had a normal neurological examination, optimal Barthel and Rankin scores, and a quality of life similar to that in a reference population. Three months postoperatively five patients $(28 \% ; 95 \%$ confidence interval (95\% CI) $10-54 \%)$ had neurological impairments (one after angiography), two $(11 \% ; 95 \%$ CI $1-35 \%)$ had a decrease in Barthel index, and $15(83 \% ; 95 \%$ CI $59-96 \%$ ) had suboptimal Rankin scores (none was dependent in daily living). Quality of life (SIP and SF-36) was reduced for most domains. After 1 year, five patients still had neurological impairments, all had an optimal Barthel index, and eight (47\%; $95 \%$ CI $23-72 \%)$ had suboptimal Rankin scores. Quality of life returned to baseline levels for all SIP and most SF-36 domains.

Conclusions-Treatment of unruptured aneurysms has a considerable short term negative impact on functional health and quality of life in most patients, despite the low rate of impairments. Outcome improves markedly but not completely within 1 year after operation.

(F Neurol Neurosurg Psychiatry 2000;68:571-576)
\end{abstract}

Keywords: aneurysm; angiography; surgery; quality of life; outcome

Because prognosis after subarachnoid haemorrhage is still poor, ${ }^{1}$ elective operation for unruptured intracranial aneurysms is often performed. Even screening for the presence of unruptured aneurysms is advocated in persons at increased risk for subarachnoid haemor- rhage such as patients with autosomal dominant polycystic kidney disease or with a family history of subarachnoid haemorrhage. ${ }^{23}$ For the assessment of the efficiency of screening with subsequent elective operation of unruptured aneurysms, the risk of rupture and subsequent subarachnoid haemorrhage has to be weighed against the risks of and prospects after elective operation. For a systematic description of outcome the World Health Organisation distinguishes three levels of health: impairments, disabilities, and handicaps. ${ }^{4}$ These measures range from being disease specific, objective, and iatrocentric (impairments) to being comprehensive, subjective, and most relevant for patients (handicap and, at the end of the range, quality of life). ${ }^{5}$ Many studies have reported on mortality and morbidity after elective operation for intracranial aneurysms. Unfortunately, outcome measures were poorly defined or restricted to neurological impairments in most of these studies. ${ }^{6}$ To our knowledge, postoperative quality of life in patients operated on for unruptured aneurysms has never been considered.

We assessed outcome in terms of impairments, disabilities, handicaps, and quality of life in patients who underwent angiography and elective clipping of asymptomatic unruptured aneurysms after participation in a screening programme for familial subarachnoid haemorrhage.

\section{Methods}

The study was approved by the ethics committees of the two participating university hospitals in The Netherlands. A consecutive series of 193 patients with subarachnoid haemorrhage admitted in these two centres between December 1995 and March 1997 was included in the study. All first degree relatives (parents, siblings, and children) between 20 and 70 years old were invited to participate in a family screening programme. Magnetic resonance angiography (MRA) was performed in 626 relatives who gave consent for screening. Eighteen of these 626 participants underwent conventional angiography to confirm the presence of one or more unruptured aneurysms, as well as subsequent elective operation.

In these 18 patients an independent physician prospectively assessed impairments, disability, handicap, and quality of life to evaluate the impact of elective operation and the preceding angiogram. Impairments were 
measured with a neurological examination on four occasions: before angiography, within 1 day after angiography, and 1 day and 3 months after operation. If neurological deficits were still present at 3 months after operation, these deficits were again examined 1 year postoperatively.

We assessed disability in activities of daily life by means of the Barthel index, ${ }^{7}$ handicap by means of the modified Rankin scale, ${ }^{89}$ and quality of life by means of the sickness impact profile $(\text { SIP })^{10}$ and medical outcomes study short form 36 (SF-36). ${ }^{11}$ The Barthel index and Rankin scale are reliable and valid instruments often used in stroke research and available in Dutch translations. ${ }^{12-14}$ The SIP is available in a validated Dutch version, ${ }^{15}$ and is a well evaluated and reliable 136 item quality of life measure, grouped into 12 domains: sleep/ rest, emotional behaviour, body care/ movement, household management, mobility, social interaction, ambulation, alertness/ behaviour, communication, work, recreation pastimes, and eating. In addition, aggregated scores can be obtained for a physical dimension subset (derived from the domains ambulation, mobility, and bodycare/movement), a psychosocial dimension subset (derived from the domains social interaction, alertness/ behaviour, emotional behaviour, and communication) and the total SIP (derived from all domains together). The SIP describes quality of life in behavioural terms and is reported to be responsive to changes over time. ${ }^{16}$ The SF-36 is a 36 item self administered quality of life measure, which includes questions on subjective health perception (by contrast with the SIP). It consists of eight domains: physical functioning, social functioning, role limitations because of physical health problems, role limitations because of emotional health problems, mental health, vitality, pain, and a single domain of general health perception. The SF-36 is available in a validated Dutch version, ${ }^{17}$ and is responsive to changes over time. ${ }^{18} 19$ The Barthel index, Rankin scale, SIP, and SF-36 were completed three times: before angiography, and 3 months (mean 3.3 months (SD) 1.2)) and 1 year (mean 14.8 months (SD 5.1)) after the operation. If a patient underwent more than one surgical procedure, follow up was performed after the last operation.

The results of neurological examination, Barthel index, and Rankin scale are presented in a descriptive manner. For the SIP and SF-36 we calculated mean scores for the 18 patients for all domains. We compared the mean preangiography scores of our study population with those of a random population reference sample. ${ }^{1517}$ We used the non-parametric Wilcoxon matched pairs test to compare the preangiography scores of all 18 patients with the scores 3 months and 1 year after operation.

\section{Results}

PATIENTS

Eighteen patients underwent angiography and intracranial operation between June 1996 and July 1998, nine in each study hospital. Eleven were women. The mean (SD) age was 44 (12) years. The mean (SD) interval between angiography and operation was 68 (50) days. Thirteen patients had one aneurysm, four had two aneurysms, and one patient had four aneurysms. The aneurysms were located at the internal carotid artery (four right, five left), the middle cerebral artery (seven right, four left), the anterior communicating artery (three), the basilar artery (one), and the pericallosal artery (one). The median diameter was $5 \mathrm{~mm}$ (range 2 to $11 \mathrm{~mm}$ ). All 25 aneurysms were clipped, apart from one entirely located in the cavernous sinus, which remained untreated. In nine patients (with 12 aneurysms) postoperative angiography was performed; in two of them an aneurysm seemed to be occluded incompletely. In the first of these two patients (with a large aneurysm of $11 \mathrm{~mm}$ ) the position and shape of the aneurysm precluded complete occlusion. The second patient underwent a second surgical procedure, which resulted in adequate clip placement, confirmed by a postoperative angiogram. Two other patients, with bilaterally located multiple aneurysms, underwent two separate operations within 1 month. The operations were done by four different neurosurgeons, all experienced in aneurysm surgery.

IMPAIRMENTS: NEUROLOGICAL EXAMINATION Before angiography all 18 patients had a normal neurological examination. During angiography (catheterisation of a vertebral artery) one patient developed drowsiness, confusion, diplopia, dysarthria, and ataxia resulting from a cerebellar and brainstem infarct (confirmed by CT). Diplopia and ataxia improved only partially, and were still present at the follow up examinations 3 months and 1 year after the operation. The remaining 17 patients had no neurological complications after angiography.

Operation resulted in persistent neurological sequelae in four patients. In all four these sequelae were present at 3 months as well as at 1 year after the operation. These sequelae were partial hemianopia (from a cerebral infarct confirmed by CT) in one patient with an aneurysm of the internal carotid artery; severe unilateral visual deficit in a second patient with an ophthalmic artery aneurysm (CT showed no abnormalities); anosmia; and a transient diplopia in a third patient who was operated on two aneurysms in a single procedure; and anosmia and a slight unilateral ptosis in the fourth patient operated on in two stages for bilaterally located aneurysms. Four other patients had transient neurological symptoms that resolved within 3 months after the operation: hemiparesis in one patient (after temporary occlusion of the middle cerebral artery during the surgical procedure), partial facial palsy and leakage of cerebrospinal fluid in another, drowsiness (from a subdural haematoma) in a third patient, and diplopia and a unilateral visual deficit in a fourth patient (after one internal carotid artery had to be occluded because of intraoperative rupture of the aneurysm). The patient with angiographic complications was operated on without additional problems. 
The combination of angiography and operation resulted in permanent (still present after 3 months and after 1 year) neurological sequelae in five of 18 patients $(28 \%$; 95\% confidence interval (95\% CI) $10-54 \%)$. If anosmia and a slight unilateral ptosis were not included, three of 18 patients ( $17 \%$; 95\% CI $4-41 \%$ ) had permanent neurological sequelae.

DISABILITY: BARTHEL INDEX

Before angiography all 18 patients had an optimal Barthel index (total score of 20). Three months after operation the Barthel index had decreased to 19 for two of the 18 patients (11\%; 95\% CI $1-35 \%)$ : one was occasionally incontinent for urine, the other needed help with bathing. One year after operation, all patients had returned to an optimal total score of 20 .

HANDICAP: RANKIN SCALE

Before angiography all 18 patients had an optimal Rankin score. Three months after operation 15 patients $(83 \%$; 95\% CI $59-96 \%)$ had a decrease in functional outcome. Ten patients had minor symptoms not interfering with lifestyle (Rankin 1), four patients had minor handicaps (Rankin 2), and one patient had a moderate handicap (Rankin 3). After 1 year we excluded one patient (with Rankin grade 1 at 3 months postoperatively), because of a newly diagnosed haematological malignancy. Eight of the remaining 17 patients $(47 \% ; 95 \%$ CI $23-72 \%$ ) still had a suboptimal Rankin score at 1 year: four had Rankin grade 1 and four had Rankin grade 2 . Comparing the results at an individual level, functional outcome had improved in eight patients, decreased in one, and remained the same in eight patients.

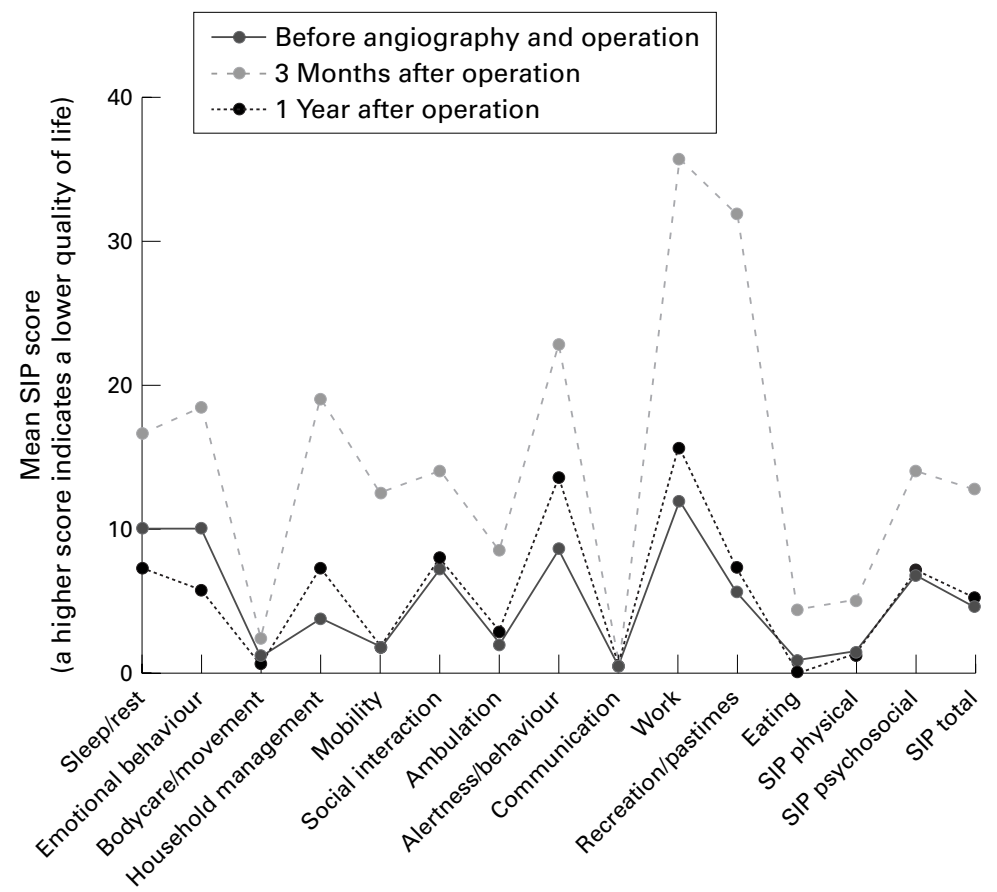

SIP domains
QUALITY OF LIFE: SIP AND SF-36

The mean SIP scores of 18 patients at the three different times are shown in fig 1 . The reference population (not shown) had scores similar to our study group before angiography, except for the domains sleep/rest and emotional behaviour, these being less favourable in our patients. Three months after operation, quality of life was lower than before angiography in all SIP domains except communication. This difference was statistically significant for the following domains: household management, mobility, social interaction, ambulation, alertness/behaviour, work, recreation pastimes, eating, and also for the SIP physical dimension subset, the SIP psychosocial subset, and the total SIP. On analysis of the aggregated "SIP total" score per individual patient, 16 patients had a lower quality of life 3 months after surgery, whereas two patients had a higher quality of life than before angiography. Of the 16 patients with a reduced quality of life, 13 had a suboptimal Rankin score, two had a suboptimal Barthel index, and four had neurological impairments. One year after operation, the mean SIP scores indicate that quality of life had considerably improved compared with the situation at 3 months postoperatively. The means of all domains had returned to preoperative values. On analysis of the "SIP total" score per individual patient, 10 patients had a lower quality of life 1 year after surgery, six patients had a higher quality of life, and in one patient quality of life was similar to preoperative values. Of the 10 patients with a reduced quality of life, six had a suboptimal Rankin score, none had a suboptimal Barthel index, and three had neurological impairments. Here also, quality of life was not assessed after 1 year in the patient with the newly diagnosed haematological malignancy.

The mean SF-36 scores of 18 patients are shown in fig 2 . The scores in the reference population were similar to those in our study group before angiography, except for the domains physical functioning and pain; in these two domains the patients had better scores than the reference population. Three months after operation, quality of life was lower than at baseline in all SF-36 domains. This difference was statistically significant for the domains: physical functioning, social functioning, physical role limit, vitality, and pain. On analysis per individual patient, in each domain an average of 11 patients showed a decrease in quality of life (the remaining patients had unchanged or better scores). One year after operation, the mean values of all domains showed an improvement in quality of life compared with the situation 3 months postoperatively. Only the domains physical role limit, vitality, and pain still showed a significant decrease compared with the preintervention scores; the scores in the remaining domains had returned to baseline levels. On analysis per individual patient, an average of eight patients per domain showed a decrease in quality of life compared with the baseline situation; the remaining nine patients had an unchanged or better quality of life. The patient with the newly 


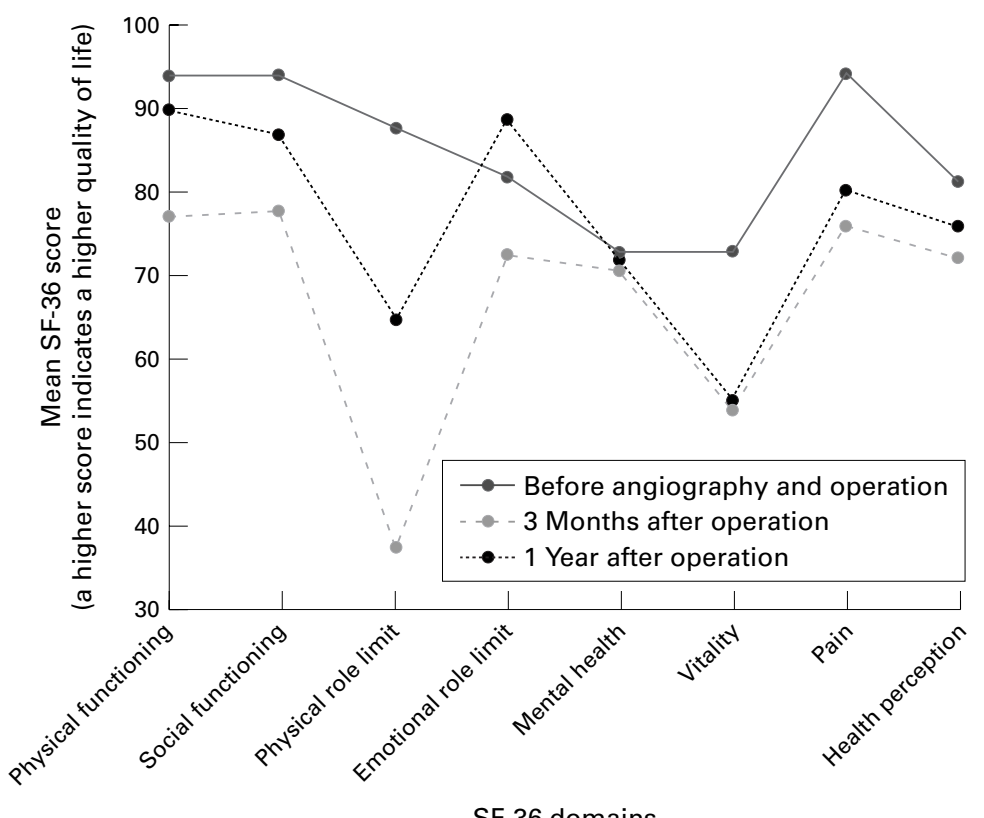

Figure 2 Mean SF-36 scores. less than optimal Rankin handicap score. One year after operation the same five patients still had neurological impairments (although these had improved), none had disabilities in self care, and eight still had a suboptimal Rankin score. The average quality of life was markedly and significantly reduced 3 months postoperatively, but after 1 year it had returned to preoperative levels for all SIP and most SF-36 domains.

We measured outcome after angiography and elective operation at four different levels of health: impairments, disabilities, handicaps, and quality of life. The neurological examination identified only a few patients with postoperative impairments, although we used strict criteria by including a relatively mild symptom of anosmia as "impairment". The Barthel index identified even fewer patients with disabilities; this confirmed a poor sensitivity for subtle changes in functional health (ceiling effect). ${ }^{12}$ By contrast, the Rankin scale indicated a deterioration in functional health for a substantially larger proportion of our study group. The SIP and SF-36 showed a reduction in quality of life for most patients. These questionnaires are likely to be more sensitive to changes in health status than the neurological examination or the Barthel index. The results of the SIP and SF-36 were similar: both quality of life measures seemed to be responsive to changes in quality of life over time, although the SIP seems to have a ceiling effect (minor changes in quality of life 1 year after operation were detected by the SF-36 but not by the SIP). We conclude that the measurement of postoperative outcome depends to a large degree on the measure of outcome used (fig 3). For a neurosurgeon, neurological impairments might seem most relevant for evaluating the outcome after operation. From a patient's perspective, however, quality of life is probably the most relevant outcome measure. Yet it takes some time to complete quality of life questionnaires (in our study an average of 30 minutes for the SIP and 15 minutes for the SF-36). The Rankin scale (1 minute), which identified most patients with a reduced quality of life, seems to be the best alternative outcome measure in everyday practice, when time is scarce..$^{20}$

Most patients with a suboptimal Rankin score or decreased quality of life did not have detectable impairments or disabilities. Most of the patients with suboptimal Rankin scores (10 of the 15 at 3 months and four of the eight at 1 year) had a score of 1 on the Rankin scale, which means minor symptoms not interfering with lifestyle. These patients often reported non-specific symptoms such as fatigue, headache, dizziness, and feelings of depression and anxiety, symptoms which probably underlie the reduction of quality of life. This finding is in keeping with another publication which concluded that patients with a score of 1 on the Rankin scale have a significant lower quality of life than patients with a Rankin score of $0 .^{20}$

The baseline quality of life of our study population was similar to that of a random population sample; only for a few aspects of quality of life were some differences found. 
According to the SIP, our patients slept worse and were more emotional, probably because they were about to undergo angiography and probably also an intracranial operation. Moreover, they all had a first degree relative with a recent episode of subarachnoid haemorrhage, of whom $25 \%$ died. According to the SF-36, our patients had a better "physical functioning" and less pain than the reference population. This may result from the exclusion of subjects with severe comorbidity from screening and treatment of aneurysms.

Outcome after angiography and operation for unruptured intracranial aneurysms does not reach a "steady state" at 3 months postoperatively. Neurological sequelae continue to improve until 1 year after operation, and possibly even longer. The same holds true for disabilities, handicaps, and especially quality of life, at least for most patients. We conclude that outcome measurement after elective treatment of unruptured aneurysms should not only be performed directly after the intervention, but also after a sufficient duration of follow up, of at least 1 year.

We identified our patients through a screening programme among first degree relatives of a consecutive series of patients with subarachnoid haemorrhage. This means that our results only apply to patients with incidentally found, mostly small aneurysms, and one relative with subarachnoid haemorrhage. It is not known whether a multiple family history of aneurysms influences outcome after elective operation. Postoperative outcome may be different in other categories of patients with unruptured aneurysms, such as those with subarachnoid haemorrhage and additional aneurysms or those with large or symptomatic aneurysms, especially if these are located at the posterior circulation.

A recent meta-analysis on operation for unruptured aneurysms found an overall mortality rate of $2.6 \%$ and morbidity rate of $10.9 \% .^{6}$ Comparison with the results of our study is difficult, because the source publications on which the overview was based were often not clear about the criteria for morbidity. Several articles included only morbidity causing dependency in daily living. Moreover, in most previous publications the rating of postoperative outcome was done by the neurosurgeon who performed the operation, a source of bias which is likely to lead to underestimation of the complication rate. In the study reported here, we used an independent observer and defined any neurological abnormality, including anosmia, as "impairment". This may have resulted in a higher, but more realistic rate of postoperative sequelae. Quality of life in our study, importantly reduced after operation for unruptured aneurysms, was not assessed in any of the previous publications.

To assess the effectiveness of screening for unruptured aneurysms, outcome after elective operation should be weighed against the risk of rupture and subsequent subarachnoid haemorrhage. The indication for screening also depends on factors such as the frequency of aneurysms in the study population, individual patient characteristics such as age and attitude towards screening, and outcome after subarachnoid haemorrhage. The results of this study on outcome after elective operation for unruptured aneurysms do not justify conclusions about the implementation of a widespread screening programme.

We conclude that elective operation for unruptured aneurysms has an important negative impact on functional health and quality of life in most patients. One year after operation, however, outcome has considerably improved, but recovery was not complete. When the pros and cons of screening for and treatment of incidental intracranial aneurysms are weighed, outcome should be assessed after 1 year, and in terms of quality of life or at least handicap.

This work was funded by a grant from the Fund of the Investigative Medicine Commission of the Dutch Health Insurance Council.

\section{Appendix}

Members of the MARS (Magnetic Resonance Angiography in Relatives of Patients with Subarachnoid Haemorrhage) Study Group:

T W M Raaymakers, MD *2,4,8,10,11,12; G J E Rinkel, $M D \star 1,2,5,8,10,11,13$; Professor C A F Tulleken, MD $+6,11$; K W Albrecht, $\mathrm{MD} \ddagger 6,11$; A Algra, MD $\star \star \star 1,2,8,10,11$; Professor P M M Bossuyt, PhD $₫ 1,2,8,9,10,11$; Professor J van Gijn, MD, FRCPE * 1,11 ; P Greebe, $\mathrm{RN} \star 4,7,10$; A Gorissen, $\mathrm{RN} \uparrow 4,7,10$; M Limburg, MD $\mid \uparrow \uparrow 1,2,5,8,10$; E Buskens, MD $\star \star 2,10$; C M Vonk, Msc $₫ 2,8,9,10 ;$ G J Bonsel, $\mathrm{MD} \$ 2,8,9,10 ; \mathrm{B}$ Verbeeten jr, MD ††3,5; F J Hulsmans, MD ††5; P C Buys,

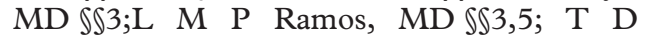
Witkamp, MD $\$ \$ 5$; Professor W P T M Mali, $\mathrm{MD} \$ \$ 1,3$

* Department of Neurology, University Hospital, Utrecht

$\dagger$ Department of Neurosurgery, University Hospital, Utrecht

‡ Department of Neurosurgery, Academic Medical Centre, Amsterdam

$\int$ Department of Clinical Epidemiology and Biostatistics, Academic Medical Centre, Amsterdam

I Department of Neurology, Academic Medical Centre, Amsterdam

$\star \star$ Julius Centre for Patient Oriented Research, University Hospital, Utrecht

t† Department of Radiology, Academic Medical Centre, Amsterdam

IS Department of Radiology, University Hospital, Utrecht

9थ Clinical Investigator of The Netherlands Heart Association

1 Design of the study and grant application

2 Development ofstudy protocol and questionnaires

3 Development of MRA protocols

4 Interviews and examinations of the participants

5 Evaluation of MRA and conventional angiograms

6 Surgical evaluation and treatment

7 Data management

8 Data analyses 
9 Cost effectiveness analyses

10 Steering committee

11 Writing committee for present manuscript

12 Study coordinator

13 Principal investigator

1 Hop JW, Rinkel GJE, Algra A, et al. Case-fatality rates and functional outcome after subarachnoid hemorrhage: a systematic review. Stroke 1997;28:660-4.

2 Ruggieri PM, Poulos N, Masaryk TJ, et al. Occult intracranial aneurysms in polycystic kidney disease: screening with MR angiography. Radiology 1994;191:33-9.

3 Bromberg JEC, Rinkel GJE, Algra A, et al. Subarachnoid hromberg JEC, Rinkel GJE, Algra A, et al. Subarachnoid haemorrhage in first and second degree relatives of patients

4 World Health Organization. International classification of impairments, disabilities and handicaps. Geneva, Switzerland: World Health Organization; 1980.

5 van Gijn J. Measurement of outcome in stroke prevention trials. Cerebrovasc Dis 1992;2(suppl 1):23-34.

6 Raaymakers TWM, Rinkel GJE, Limburg $\mathrm{M}$, et al. Mortality and morbidity of surgery for unruptured intracranial aneurysms: a meta-analysis. Stroke 1998;29:1531-8.

7 Mahoney FI, Barthel DW. Functional evaluation: the Barthel index. Md State Med $\mathcal{F}$ 1965;14:61-5.

8 Rankin J. Cerebral vascular accidents in people over the age of 60. II. Prognosis. Scot Med f 1957;2:200-15.

9 Bamford JM, Sandercock PAG, Warlow CP, et al. Interobserver agreement for the assessment of handicap in stroke

10 Bergner M, Bobbitt RA, Carter WB, et al. The sickness impact profile: development and final revision of a healthstatus measure. Med Care 1981;19:787-805.
11 Ware JE Jr, Sherbourne CD. The MOS 36-item short-form health survey (SF-36). I. Conceptual framework and item selection. Med Care 1992;30:473-83.

12 de Haan R, Limburg M, Schuling J, et al. Clinimetric evaluation of the Barthel index, a measure of limitations in daily activities. Ned Tijdschr Geneeskd 1993;137:917-21.

13 Wolfe CD, Taub NA, Woodrow EJ, et al. Assessment of scales of disability and handicap for stroke patients. Stroke 1991;22:1242-4.

14 van Swieten JC, Koudstaal PJ, Visser MC, et al. Interobserver agreement for the assessment of handicap in stroke patients. Stroke 1988;19:604-7.

15 Jacobs HM, Luttik A, Touw Otten FW, et al. The sickness impact profile; results of an evaluation study of the Dutch version. Ned Tijdschr Geneeskd 1990;134:1950-4.

16 de Bruin AF, Diederiks JP, de Witte LP, et al. Assessing the responsiveness of a functional status measure: the sickness impact profile versus the SIP68. F Clin Epidemiol 1997;50: $529-40$

17 van der Zee K, Sanderman R, Heyink J. De psychometrische kwaliteiten van de MOS 36-item short form health survey (SF-36) in een Nederlandse populatie. Tgdschrift voor Sociale Gezondheidszorg 1993;71:183-91.

18 Mangione CM, Goldman L, Orav EJ, et al. Health-related quality of life after elective surgery: measurement of quality of life after elective surgery: measurement of long.

19 Jenkinson C, Lawrence K, McWhinnie D, et al. Sensitivity to change of health status measures in a randomized controlled trial: comparison of the COOP charts and the SF-36. Oual Life Res 1995;4:47-52.

20 Hop JW, Rinkel GJE, Algra A, et al. Quality of life in patients and partners after aneurysmal subarachnoid hemorrhage. Stroke 1998;29:798-804. 